International Mathematical Forum, 1, 2006, no. 29, 1423 - 1432

\title{
Testing the Equality of a Set of Multivariate Linear Models
}

\author{
José A. Díaz-García \\ Department of Statistics and Computation \\ Universidad Autónoma Agraria Antonio Narro \\ 25350 Buenavista, Saltillo, Coahuila, MÉXICO \\ jadiaz@uaaan.mx \\ Francisco José Caro-Lopera \\ Department of Basic Mathematics \\ Centro de Investigación en Matemáticas \\ Callejón de Jalisco s/n, 36000 Guanajuato, Gto., México \\ fjcaro@cimat.mx
}

\begin{abstract}
This work derives a procedure for testing the hypothesis about the equality among $T$ multivariate linear models. The results are extended to the case where the errors follow an elliptic distribution
\end{abstract}

Keywords: Multivariale linear model, elliptical distributions, likelihood ratio test, union-intersection criterion, Pillai's trace, Lawlwy-Hotelling trace.

Mathematics Subject Classification: 62H15, 62H10

\section{INTRODUCTION}

The general multivariate linear model can be written as follows

$$
\mathbf{y}_{i}=\mathbf{X}_{i} \boldsymbol{\beta}_{i}+\boldsymbol{\epsilon}_{i}, \quad i=1, \ldots, p
$$

where $\mathbf{y}_{i} \in \Re^{n_{i} \times 1}, \mathbf{X}_{i} \in \Re^{n_{i} \times q_{i}}, \boldsymbol{\beta}_{i} \in \Re^{q_{i} \times 1}$ and under the normal theory, $\boldsymbol{\epsilon}_{i} \sim \mathcal{N}_{n_{i}}\left(\mathbf{0}, \Sigma_{i}\right)$. Several particular cases of that model have been studied in the literature. For example, when $n_{i}=N$ and $\Sigma_{i}=\Sigma$ for every $i=1, \ldots, p$, it is known as seemingly unrelated regression model and it was treated by Zellner (1962) (also see Press (1982, Section 8.5.1, p. 239)). Another special models of concern in statistical literature were given by Zellner (1962) and they can be found when: 
- $\boldsymbol{\beta}_{1}=\boldsymbol{\beta}_{2}=\cdots=\boldsymbol{\beta}_{p}$ and the matrices $\mathbf{X}_{1}, \mathbf{X}_{2}, \ldots, \mathbf{X}_{p}$ are unequal;

- $\boldsymbol{\beta}_{1}=\boldsymbol{\beta}_{2}=\cdots=\boldsymbol{\beta}_{p}$ and $\mathbf{X}_{1}=\mathbf{X}_{2}=\cdots=\mathbf{X}_{p}$.

Theory and applications of the last two models are exposed in Box and Tiao (1972, Chapter 9, p. 478).

The most well known particular model in the literature it is obtained from (1) by taking $q_{i}=q, n_{i}=n, \mathbf{X}_{i}=\mathbf{X}$ and $\Sigma_{i}=\Sigma$, with $i=1, \ldots, p$. Thus (1) becomes

$$
\mathbf{Y}=\mathbf{X} \boldsymbol{\beta}+\boldsymbol{\epsilon}
$$

where, $\mathbf{Y}=\left(\mathbf{y}_{1} \cdots \mathbf{y}_{p}\right) \in \Re^{n \times p}, \boldsymbol{\beta}=\left(\boldsymbol{\beta}_{1} \cdots \boldsymbol{\beta}_{p}\right) \in \Re^{q \times p}, \boldsymbol{\epsilon}=\left(\boldsymbol{\epsilon}_{1} \cdots \boldsymbol{\epsilon}_{p}\right) \sim$ $\mathcal{N}_{n \times p}\left(\mathbf{0}, \mathbf{I}_{n} \otimes \boldsymbol{\Sigma}\right)$, and $\otimes$ denotes the Kronecker product. Besides, if $q+p \leq n$, then the likelihood maximum estimators for the parameters $\boldsymbol{\beta}$ and $\boldsymbol{\Sigma}$ are given by

$$
\begin{aligned}
\widetilde{\boldsymbol{\beta}} & =\left(\mathbf{X}^{\prime} \mathbf{X}\right)^{-1} \mathbf{X}^{\prime} \mathbf{Y}=\mathbf{X}^{-} \mathbf{Y} \\
\widetilde{\boldsymbol{\Sigma}} & =\frac{1}{n}(\mathbf{Y}-\mathbf{X} \widetilde{\boldsymbol{\beta}})^{\prime}(\mathbf{Y}-\mathbf{X} \widetilde{\boldsymbol{\beta}}),
\end{aligned}
$$

respectively; where $\mathbf{X}^{-}$is the Moore-Penrose inverse of $\mathbf{X}$; see Roy (1957), Morrison (1982), Press (1982), Muirhead (1982), Seber (1984) and Rencher (1995), among many others. For different situations, it becomes of interest verifying if the multivariate linear models are equal, when those ones are proposed to model the same situation under different conditions. For example: Suppose $p$ dependent variables $Y_{1}, Y_{2}, \ldots, Y_{p}$, which are funtions of $q$ independent variables $X_{1}, X_{2}, \ldots, X_{q}$, will be measured in $n$ individuals, and the model to follow has the form (2). Besides, let us suppose that the above situation is presented in $T$ different conditions (they could be $T$ conditions, $T$ different places, $T$ different temperatures, etc. ), but, the remaining factors among the different conditions are homogeneous. So, a question to solve talks about if the dependent variables $Y_{1}, Y_{2}, \ldots, Y_{p}$ have the same behavior under the $T$ different conditions and under different levels in the independent variables $X_{1}, X_{2}, \ldots, X_{q}$. Rigourously, this situation can expressed as follows: let

$$
\mathbf{Y}_{t}=\mathbf{X}_{t} \boldsymbol{\beta}_{t}+\boldsymbol{\epsilon}_{t}, \quad t=1,2, \ldots, T,
$$

be multivariate linear models, where $\mathbf{Y}_{t} \in \Re^{n_{t} \times p}, \mathbf{X}_{t} \in \Re^{n_{t} \times q}$ of rank $q, \boldsymbol{\beta}_{t} \in$ $\Re^{q \times p}$ and $\boldsymbol{\epsilon}_{t} \sim \mathcal{N}_{n_{t} \times p}\left(\mathbf{0}, \mathbf{I}_{n_{t}} \otimes \boldsymbol{\Sigma}\right), \boldsymbol{\Sigma}>\mathbf{0}$. It is the objective to test the hypothesis

$$
H_{0}: \boldsymbol{\beta}_{1}=\underset{\boldsymbol{\beta}_{2}}{ }=\cdots=\boldsymbol{\beta}_{T}
$$

$H_{a}:$ at last one equality is an inequality 
In the univariate case, $p=1$, it was studied by Graybill (1976, Section 8.6.2, pp. 291-297) and Draper and Smith (1981), among others. The present work propose several statistics for testing the hypothesis (6) under the conditions of the model (5), see Section 2. The paper ends showing an example as application.

\section{TEST STATISTICS}

By mixing the conditions of the models (1) and (2), in this section are derived several statistics for testing the hypothesis which establishes that the $T$ multivariate linear models are equal.

Theorem 1 Given the model (5), the likelihood ratio test of $H_{0}: \boldsymbol{\beta}_{1}=\boldsymbol{\beta}_{2}=$ $\cdots=\boldsymbol{\beta}_{T}$ is given by

$$
\Lambda=\frac{\left|\mathbf{S}_{E}\right|}{\left|\mathbf{S}_{E}+\mathbf{S}_{H}\right|}
$$

which is termed Wilks's $\Lambda$ or it has also been termed Wilks's $U$. Where

$$
\begin{aligned}
& \mathbf{S}_{E}=\sum_{t=1}^{T} \mathbf{Y}_{t}^{\prime}\left(\mathbf{I}_{n_{t}}-\mathbf{X}_{t} \mathbf{X}_{t}^{-}\right) \mathbf{Y}_{t} \in \Re^{p \times p} \\
& \mathbf{S}_{H}=\sum_{t=1}^{T} \mathbf{Y}_{t}^{\prime}\left(\mathbf{X}_{t} \mathbf{X}_{t}^{-}\right) \mathbf{Y}_{t}-\left(\sum_{i=1}^{T} \mathbf{Y}_{i}^{\prime} \mathbf{X}_{i}\right)\left(\sum_{t=1}^{T} \mathbf{X}_{t}^{\prime} \mathbf{X}_{t}\right)^{-1}\left(\sum_{j=1}^{T} \mathbf{X}_{j}^{\prime} \mathbf{Y}_{j}\right) \in \Re^{p \times p} .(9)
\end{aligned}
$$

We reject $H_{0}$ if

$$
\Lambda \leq \Lambda_{\alpha, p, \nu_{H}, \nu_{E}}
$$

where $\nu_{H}=(T-1) q, \nu_{E}=N-T q, N=\sum_{t=1}^{T} n_{t}$. Exact critical values of $\Lambda_{\alpha, p, \nu_{H}, \nu_{E}}$ for Wilks's $\Lambda$ are found in Rencher (1995, Table A.9) or Kres (1983, Table 1).

Proof. If we write

$$
\mathbb{Y}=\left[\begin{array}{c}
\mathbf{Y}_{1} \\
\mathbf{Y}_{2} \\
\vdots \\
\mathbf{Y}_{T}
\end{array}\right], \mathbb{X}=\left[\begin{array}{cccc}
\mathbf{X}_{1} & 0 & \cdots & 0 \\
0 & \mathbf{X}_{2} & \cdots & 0 \\
\vdots & \vdots & \ddots & \vdots \\
0 & 0 & \cdots & \mathbf{X}_{T}
\end{array}\right], \mathbb{B}=\left[\begin{array}{c}
\boldsymbol{\beta}_{1} \\
\boldsymbol{\beta}_{2} \\
\vdots \\
\boldsymbol{\beta}_{T}
\end{array}\right] \text { and } \mathbb{E}=\left[\begin{array}{c}
\boldsymbol{\epsilon}_{1} \\
\boldsymbol{\epsilon}_{2} \\
\vdots \\
\boldsymbol{\epsilon}_{T}
\end{array}\right]
$$

with $\mathbb{Y} \in \Re^{N \times p}, \mathbb{X} \in \Re^{N \times T q}$ and $\mathbb{B} \in \Re^{T q \times p}$ and noting that $\mathbb{E} \sim \mathcal{N}_{N \times p}\left(\mathbf{I}_{N} \otimes\right.$ $\boldsymbol{\Sigma}), \quad N=\sum_{t=1}^{T} n_{t}$; then the $T$ models $(5)$ can be written as

$$
\mathbb{Y}=\mathbb{X B}+\mathbb{E}
$$


this is a general multivariate linear model of type (2). By noting that the hypothesis $H_{0}: \boldsymbol{\beta}_{1}=\boldsymbol{\beta}_{2}=\cdots=\boldsymbol{\beta}_{T}$ can be expressed as $\mathbf{C B}=0$, with

$$
\mathbf{C}=\left(\begin{array}{cccccc}
\mathbf{I}_{q} & -\mathbf{I}_{q} & 0 & \cdots & 0 & 0 \\
0 & \mathbf{I}_{q} & -\mathbf{I}_{q} & \cdots & 0 & 0 \\
\vdots & \vdots & \vdots & \ddots & \vdots & \vdots \\
0 & 0 & 0 & \cdots & \mathbf{I}_{q} & -\mathbf{I}_{q}
\end{array}\right) \in \Re^{(T-1) q \times T q}
$$

of rank $(T-1) q$; then, it is possible to extend the theory of the model (2) to the model (10). Like this, by Rencher (1995, p. 161), Seber (1984, p. 412) or Muirhead (1982, sections 10.1 and 10.2), among many others, likelihood ratio test is given by

$$
\Lambda=\frac{\left|\mathbf{S}_{E}\right|}{\left|\mathbf{S}_{E}+\mathbf{S}_{H}\right|}
$$

where

$$
\begin{aligned}
\mathbf{S}_{E} & =\mathbb{Y}^{\prime}\left(\mathbf{I}_{N}-\mathbb{X} \mathbb{X}^{-}\right) \mathbb{Y} \\
\mathbf{S}_{H} & =(\mathbf{C B})^{\prime}\left(\mathbf{C}\left(\mathbb{X}^{\prime} \mathbb{X}\right)^{-1} \mathbf{C}^{\prime}\right)^{-1}(\mathbf{C B})
\end{aligned}
$$

besides, by $(3), \widehat{\mathbb{B}}=\mathbb{X}^{-} \mathbb{Y}=\left(\mathbb{X}^{\prime} \mathbb{X}\right)^{-1} \mathbb{X}^{\prime} \mathbb{Y}$. But, note that

$$
\widehat{\mathbb{B}}=\left[\begin{array}{c}
\widehat{\boldsymbol{\beta}}_{1} \\
\widehat{\boldsymbol{\beta}}_{2} \\
\vdots \\
\widehat{\boldsymbol{\beta}}_{T}
\end{array}\right]=\left[\begin{array}{cccc}
\left(\mathbf{X}^{\prime} \mathbf{X}\right)_{1}^{-1} & 0 & \cdots & 0 \\
0 & \left(\mathbf{X}^{\prime} \mathbf{X}\right)_{2}^{-1} & \cdots & 0 \\
\vdots & \vdots & \ddots & \vdots \\
0 & 0 & \cdots & \left(\mathbf{X}^{\prime} \mathbf{X}\right)_{T}^{-1}
\end{array}\right]\left[\begin{array}{c}
\mathbf{X}_{1}^{\prime} \mathbf{Y}_{1} \\
\mathbf{X}_{2}^{\prime} \mathbf{Y}_{2} \\
\vdots \\
\mathbf{X}_{T}^{\prime} \mathbf{Y}_{T}
\end{array}\right]
$$

This is, $\widehat{\boldsymbol{\beta}}_{t}=\mathbf{X}_{t}^{-} \mathbf{Y}_{t}=\left(\mathbf{X}_{t}^{\prime} \mathbf{X}\right)_{t}^{-1} \mathbf{X}_{t}^{\prime} \mathbf{Y}_{t}, \quad t=1,2, \ldots, T$. Thus $\widehat{\boldsymbol{\beta}}_{t}$ is the same as if it was obtained from thr $t$ th model $\mathbf{Y}_{t}=\mathbf{X}_{t} \boldsymbol{\beta}_{t}+\boldsymbol{\epsilon}_{t}$. Now, by (4), and observing that

$$
\mathbb{Y}^{\prime} \mathbb{Y}=\sum_{t=1}^{T} \mathbf{Y}_{t}^{\prime} \mathbf{Y}_{t} \text { and } \widehat{\mathbb{B}}^{\prime} \mathbb{X}^{\prime} \mathbb{Y}=\sum_{t=1}^{T} \widehat{\boldsymbol{\beta}}_{t}^{\prime} \mathbf{X}_{t}^{\prime} \mathbf{Y}_{t}=\sum_{t=1}^{T} \mathbf{Y}_{t}^{\prime} \mathbf{X}_{t}^{\prime} \mathbf{X}_{t}^{-} \mathbf{Y}_{t}
$$

it is gotten

$$
\mathbf{S}_{E}=\sum_{t=1}^{T} \mathbf{Y}_{t}^{\prime}\left(\mathbf{I}_{n_{t}}-\mathbf{X}_{t} \mathbf{X}_{t}^{-}\right) \mathbf{Y}_{t}
$$

Under the null hypothesis, it is obtained the reduced model, $\mathbb{Y}=\mathbb{X}(\mathbf{1} \otimes \boldsymbol{\eta})+\mathbb{E}$, where $\boldsymbol{\eta}$ is the common unknown parameter matrix, $\boldsymbol{\eta}=\boldsymbol{\beta}_{1}=\cdots=\boldsymbol{\beta}_{T}$ and $\mathbf{1}=(1, \ldots, 1)^{\prime} \in \Re^{T}$. Taking in count that for $\mathbb{B}$ (or $\boldsymbol{\eta}$ ) its likelihood maximum 
estimator coincides with its minimum squared estimator, we can proceed as follows. Let

$$
\begin{aligned}
Q & =\min _{\eta} \operatorname{tr}\left(\mathbb{E}^{\prime} \mathbb{E}\right) \\
& =\min _{\eta} \operatorname{tr}\left(\sum_{i=1}^{T} \boldsymbol{\epsilon}_{t}^{\prime} \boldsymbol{\epsilon}_{t}\right) \\
& =\min _{\eta} \operatorname{tr}\left(\sum_{i=1}^{T}\left(\mathbf{Y}_{t}-\mathbf{X}_{t} \boldsymbol{\eta}\right)^{\prime}\left(\mathbf{Y}_{t}-\mathbf{X}_{t} \boldsymbol{\eta}\right)\right) \\
& =\min _{\eta} \operatorname{tr}\left(\sum_{i=1}^{T} \mathbf{Y}_{t}^{\prime} \mathbf{Y}_{t}-2\left(\sum_{i=1}^{T} \mathbf{Y}_{t}^{\prime} \mathbf{X}_{t}\right) \boldsymbol{\eta}+\boldsymbol{\eta}\left(\sum_{i=1}^{T} \mathbf{X}_{t} \mathbf{X}_{t}\right) \boldsymbol{\eta}\right)
\end{aligned}
$$

where $\operatorname{tr}(\cdot)$ denote the trace. Thus

$$
\widehat{\boldsymbol{m}}=\left(\sum_{i=1}^{T} \mathbf{X}_{t} \mathbf{X}_{t}\right)^{-1}\left(\sum_{i=1}^{T} \mathbf{X}_{t}^{\prime} \mathbf{Y}_{t}\right)
$$

Then

$$
\begin{aligned}
\mathbf{S}_{H} & =\mathbb{Y}^{\prime} \mathbb{Y}-\widehat{\boldsymbol{\eta}}^{\prime} \mathbb{X}^{\prime} \mathbb{Y}-\mathbf{S}_{E} \\
& =\sum_{t=1}^{T} \mathbf{Y}_{t}^{\prime}\left(\mathbf{X}_{t} \mathbf{X}_{t}^{-}\right) \mathbf{Y}_{t}-\left(\sum_{i=1}^{T} \mathbf{Y}_{i}^{\prime} \mathbf{X}_{i}\right)\left(\sum_{t=1}^{T} \mathbf{X}_{t}^{\prime} \mathbf{X}_{t}\right)^{-1}\left(\sum_{j=1}^{T} \mathbf{X}_{t}^{\prime} \mathbf{Y}_{j}\right)
\end{aligned}
$$

and the desired result is obtained.

Alternatively we get.

Theorem 2 Given the model (5), the union-intersection test of $H_{0}: \boldsymbol{\beta}_{1}=$ $\boldsymbol{\beta}_{2}=\cdots=\boldsymbol{\beta}_{T}$ is given by

$$
\theta=\frac{\lambda_{1}}{1+\lambda_{1}}
$$

which is termed Roy's largest root test. Where $\lambda_{1}$ is the maximum eigenvalue of $\left(\mathbf{S}_{H} \mathbf{S}_{E}^{-1}\right)$, where $\mathbf{S}_{H}$ and $\mathbf{S}_{E}$ are given by (9) and (8), respectively. We reject $H_{0}$ if $\theta \geq \theta_{\alpha, s, m, h}$. The parameters $s, m$ and $h$ are defined as

$$
s=\min \left(p, \nu_{H}\right), \quad m=\left(\left|p-\nu_{H}\right|-1\right) / 2, \quad h=\left(\nu_{E}-p-1\right) / 2 .
$$

As in Theorem 1, $\nu_{H}=(T-1) q, \nu_{E}=N-T q$ and $N=\sum_{t=1}^{T} n_{t}$. Exact critical values of $\theta_{\alpha, s, m, h}$ are found in Rencher (1995, Table A.10) or Kres (1983, Tables 2, 4 and 5). 
Proof. We proceed as in Theorem 1: by (10) and (11), the multivariate hypothesis can be expressed as

$$
H_{0}: \mathbf{C B}=\mathbf{0}
$$

which is true if and only if the univariate hypotheses

$$
H_{0 \mathbf{a}}: \mathbf{C B} \mathbf{a}=0
$$

hold for all non-null vectors a. The statistic for all the univariate hypothesis is given by

$$
F(\mathbf{a})=\frac{(N-T q) \sum_{t=1}^{T} \mathbf{a}^{\prime} \mathbf{Y}_{t}^{\prime}\left(\mathbf{X}_{t} \mathbf{X}_{t}^{-}\right) \mathbf{Y}_{t} \mathbf{a}-\mathbf{a}^{\prime}\left(\sum_{i=1}^{T} \mathbf{Y}_{i}^{\prime} \mathbf{X}_{i}\right)\left(\sum_{t=1}^{T} \mathbf{X}_{t}^{\prime} \mathbf{X}_{t}\right)^{-1}\left(\sum_{j=1}^{T} \mathbf{X}_{j}^{\prime} \mathbf{Y}_{j}\right) \mathbf{a}}{(T-1) q \sum_{t=1}^{T} \mathbf{a}^{\prime} \mathbf{Y}_{t}^{\prime}\left(\mathbf{I}_{n_{t}}-\mathbf{X}_{t} \mathbf{X}_{t}^{-}\right) \mathbf{Y}_{t} \mathbf{a}}
$$

where $N=\sum_{t=1}^{T} n_{t}$, see Graybill (1976, Theorem 8.6.4, p. 291). For an univariate test of confidence level $\gamma, H_{0 \mathbf{a}}: \mathbf{C B} \mathbf{a}=0$ is accepted if

$$
F(\mathbf{a}) \leq F_{\gamma,(T-1) q, N-T q}
$$

where $F_{\gamma,(T-1) q, N-T q}$ is the upper $\gamma$ probability point of the central $F$-distribution with $(T-1) q$ and $N-T q$ degrees freedom. So, proceeding as in Roy (1957, Section 12.7, pp. 82 -83) (also see Morrison (1982, pp. 176-177)), we have for $H_{0}=\bigcap_{\mathbf{a}} H_{0 \mathbf{a}}$, the critical region of size $\alpha(>\gamma)$ is given by

$$
\bigcap_{\mathbf{a}}\left[F(\mathbf{a}) \leq F_{\gamma,(T-1) q, N-T q}\right]
$$

which is equivalent to that defined by

$$
\max _{\mathbf{a}} F(a) \leq F_{\gamma,(T-1) q, N-T q}
$$

over all non-null a. This way we reject $H_{0}$ if

$$
\theta \geq \theta_{\alpha, s, m, h}
$$

with

$$
s=\min (p,(T-1) q), \quad m=(|p-(T-1) q|-1) / 2, \quad h=(N-T q-p-1) / 2, \quad N=\sum_{t=1}^{T} n_{t} .
$$


where $\theta=\lambda_{1} /\left(1+\lambda_{1}\right), \lambda_{1}$ is the maximum eigenvalue of $\left(\mathbf{S}_{H} \mathbf{S}_{E}^{-1}\right)$ and

$$
\begin{aligned}
\mathbf{S}_{E} & =\sum_{t=1}^{T} \mathbf{Y}_{t}^{\prime}\left(\mathbf{I}_{n t}-\mathbf{X}_{t} \mathbf{X}_{t}^{-}\right) \mathbf{Y}_{t} \\
\mathbf{S}_{H} & =\sum_{t=1}^{T} \mathbf{Y}_{t}^{\prime}\left(\mathbf{X}_{t} \mathbf{X}_{t}^{-}\right) \mathbf{Y}_{t}-\left(\sum_{i=1}^{T} \mathbf{Y}_{i}^{\prime} \mathbf{X}_{i}\right)\left(\sum_{t=1}^{T} \mathbf{X}_{t}^{\prime} \mathbf{X}_{t}\right)^{-1}\left(\sum_{j=1}^{T} \mathbf{X}_{j}^{\prime} \mathbf{Y}_{j}\right)
\end{aligned}
$$

also see Rencher (1995, Section 6.1.4, p. 164), among many others.

A lot of different test statistics have been proposed for verifying hypothesis of the kind (6). Before to show some of then, let us considerer the following notation: given $s=\min \left(p, \nu_{H}\right)$, let $\lambda_{1}, \ldots \lambda_{s}$ be the eigenvalues of the matrix $\left(S_{H} S_{E}^{-1}\right)$ such that $\lambda_{1} \geq \cdots \geq \lambda_{s}>0$ and $\theta_{1}, \ldots \theta_{s}$ the eigenvalues of the matrix $\left(S_{H}\left(S_{E}+S_{H}\right)^{-1}\right)$, with $1 \geq \theta_{1} \geq \cdots \geq \theta_{s}>0$. Observe that $\theta_{i}=\lambda_{i} /\left(1+\lambda_{i}\right)$, and, $\lambda_{i}=\theta_{i} /\left(1-\theta_{i}\right), i=1, \ldots, s$. Thus, the statistic $\Lambda$ of Wilks can be written as:

$$
\Lambda=\frac{\left|\mathbf{S}_{E}\right|}{\left|\mathbf{S}_{E}+\mathbf{S}_{H}\right|}=\prod_{i=1}^{s} \frac{1}{1+\lambda_{i}}=\prod_{i=1}^{s}\left(1-\theta_{i}\right)
$$

from where it is followed that, the range of $\Lambda$ is $0 \leq \Lambda \leq 1$. Two of these additional test statistics for the hypothesis $H_{0}: \boldsymbol{\beta}_{1}=\boldsymbol{\beta}_{2}=\cdots=\boldsymbol{\beta}_{T}$ are the following:

1. Pillai Test. The Pillai statistics is defined as

$$
V^{(s)}=\operatorname{tr}\left[\mathbf{S}_{H}\left(\mathbf{S}_{E}+\mathbf{S}_{H}\right)^{-1}\right]=\sum_{i=1}^{s} \frac{\lambda_{i}}{1+\lambda_{i}}=\sum_{i=1}^{s} \theta_{i}
$$

This way we reject $H_{0}$ if

$$
V^{(s)} \geq V_{\alpha, s, m, h}^{(s)}
$$

with $s=\min (p,(T-1) q), m=(|p-(T-1) q|-1) / 2, h=(N-T q-$ $p-1) / 2, N=\sum_{t=1}^{T} n_{t}$, and where the exact critical values of $V_{\alpha, s, m, h}^{(s)}$ are found in Rencher (1995, Table A.11) or Kres (1983, Table 7).

2. Lawley-Hotelling Test. The Lawley-Hotelling statistics is given by

$$
U^{(s)}=\operatorname{tr}\left[\mathbf{S}_{H} \mathbf{S}_{E}^{-1}\right]=\sum_{i=1}^{s} \lambda_{i}=\sum_{i=1}^{s} \frac{\theta_{i}}{1-\theta_{i}} .
$$

We reject $H_{0}$ if

$$
U^{(s)} \geq U_{\alpha, s, m, h}^{(s)}
$$


with $s=\min (p,(T-1) q), m=(|p-(T-1) q|-1) / 2, h=(N-T q-$ $p-1) / 2, N=\sum_{t=1}^{T} n_{t}$.

The upper percentage points, $U_{\alpha, s, m, h}^{(s)}$, are given in Kres (1983, Table 6). A variant of this statistics and its corresponding exact critical values are given in Rencher (1995, p. 167 and Table A.12, respectively).

Finally, note that by the theorems 5.3.3 and 5.3.4 of Gupta and Varga (1993, pp. 185-186), the four above-mentioned test statistics are invariant under the hole family of elliptical distributions, more over, their distribution coincide under normality assumption.

\section{EXAMPLE}

The following example was taken from Graybill (1976, p. 295) and it have been modified by adding a new depend variable $Y_{2}$ by simulation.

A new food supplement ( $x$ unit) was fed to three different breeds of chickens for six weeks to determine the effect on hardness $Y_{1}$ and weight $Y_{2}$ (gr.) of eggs. A simple linear multivariate model was assumed for each breed.

$$
\mathbf{Y}_{t}=\mathbf{X}_{t} \boldsymbol{\beta}_{t}+\boldsymbol{\epsilon}_{t}, \quad t=1,2,3
$$

$\boldsymbol{\epsilon}_{t} \sim \mathcal{N}_{n_{t} \times 2}\left(\mathbf{0}, \mathbf{I}_{n_{t}} \otimes \boldsymbol{\Sigma}\right), \boldsymbol{\Sigma} \in \Re^{2 \times 2}, \boldsymbol{\Sigma}>\mathbf{0}$, with $n_{1}=12, n_{2}=8$ and $n_{3}=9$ and

$$
\boldsymbol{\beta}_{t}=\left(\begin{array}{cc}
\beta_{01 t} & \beta_{02 t} \\
\beta_{11 t} & \beta_{12 t}
\end{array}\right)
$$

The problem is to determine if the models are the same for all breeds, that is, to test the hypothesis

$$
H_{0}:\left(\begin{array}{ll}
\beta_{011} & \beta_{021} \\
\beta_{111} & \beta_{121}
\end{array}\right)=\left(\begin{array}{ll}
\beta_{012} & \beta_{022} \\
\beta_{112} & \beta_{122}
\end{array}\right)=\left(\begin{array}{ll}
\beta_{013} & \beta_{023} \\
\beta_{113} & \beta_{123}
\end{array}\right)
$$

The data are given next in Table 1. 


\begin{tabular}{||rrr|rrr|rrr||}
\hline \hline \multicolumn{3}{|c|}{ Breed 1 } & \multicolumn{3}{|c|}{ Breed 2 } & \multicolumn{3}{c||}{ Breed 3 } \\
$X$ & $Y_{1}$ & $Y_{2}$ & $X$ & $Y_{1}$ & $Y_{2}$ & $X$ & $Y_{1}$ & $Y_{2}$ \\
\hline \hline 1 & 8.42 & 74.2 & 3 & 9.86 & 72.5 & 2 & 6.52 & 70.1 \\
3 & 14.68 & 69.1 & 3 & 9.54 & 71.2 & 5 & 5.11 & 72.2 \\
5 & 21.42 & 63.5 & 4 & 11.96 & 69.8 & 7 & 7.75 & 69.3 \\
6 & 25.45 & 62.8 & 5 & 12.46 & 67.8 & 8 & 6.84 & 68.5 \\
7 & 27.14 & 60.0 & 6 & 11.38 & 66.5 & 10 & 7.65 & 66.9 \\
8 & 30.53 & 57.1 & 8 & 14.69 & 62.3 & 15 & 9.49 & 63.2 \\
9 & 34.51 & 55.2 & 9 & 16.48 & 60.1 & 16 & 7.03 & 61.4 \\
9 & 34.52 & 54.9 & 12 & 20.11 & 55.7 & 18 & 9.41 & 59.7 \\
10 & 33.24 & 53.6 & & & & 20 & 12.01 & 55.6 \\
11 & 39.63 & 50.4 & & & & & & \\
12 & 43.98 & 47.3 & & & & & & \\
14 & 47.77 & 44.4 & \multicolumn{1}{|c|}{} & & & & \\
\hline
\end{tabular}

By (8) and (9), we have that

$$
\mathbf{S}_{E}=\left(\begin{array}{ll}
39326.040 & 39837.725 \\
39837.725 & 19800.195
\end{array}\right) \text { and } \mathbf{S}_{H}=\left(\begin{array}{rr}
4145.392 & -8404.961 \\
-8404.961 & 23839.162
\end{array}\right)
$$

then, the following results are obtained.

Table 1: Four criteria to proof the null hypothesis

\begin{tabular}{lll}
\hline Criteria & Statistic & $\alpha$ Critical value \\
\hline Wilks $^{a}$ & $5.856 \mathrm{e}-006$ & 0.515922 \\
Roy & 0.99977 & 0.415 \\
Pillai & 1.97383 & 0.532 \\
Lawley-Hotelling & 11721.5216 & $2.16811^{b}$ \\
\hline
\end{tabular}

\footnotetext{
${ }^{a}$ Remember that for this tests, the decision rule is: statistics $\leq$ critical value
}

${ }^{b}$ Using an F approximation, see equation (6.26) in Rencher (1995, p.166, 1995). 
It is clear that the four criterions reject the above-mentioned hypothesis.

\section{ACKNOWLEDGMENT}

This work was supported partially by the research project $45974 \mathrm{~F}$ of CONACYT-México.

\section{REFERENCES}

G. E. Box and G. C. Tiao, Bayesian Inference in Statistical Analysis, AddisonWesley, Reading, PA, 1972.

N. R. Draper and H. Smith, Applied regression analysis, 2nd edition. Wiley, New York, 1981.

F. A. Graybill, Theory and application of the linear model, Wadsworth \& Brooks/Cole, Advances Books and Softwere, Pacific Grove, California, 1976.

A. K. Gupta and T. Varga, Elliptically Contoured Models in Statistics, Kluwer Academic Publishers, Dordrecht, 1993.

H. Kres, Statistical Tables for Multivariate Analysis, Springer- Verlag, New York, 1983.

D. F. Morrison, Multivariate Sattistical Methods, McGraw Hill, London, 1978.

R. J. Muirhead, Aspects of Multivariate Statistical Theory, John Wiley \& Sons, New York, 1982.

S. J. Press, Applied Multivariate Analysis: Using Bayesian and Frequentist Methods of Inference, Second Edition, Robert E. Krieger Publishing Company, Malabar, FL, 1982.

A. C. Rencher, Methods of Multivariate Analysis, John Wiley \& Sons, New York, 1995.

S. N. Roy Some Aspect of Multivariate Analysis, John Wiley \& Sons, New York, 1957.

G. A. F. Seber, Multivariate Observations. John Wiley \& Sons, New York, 1984.

A. Zellner, An eficient method of estimating seemingly unrelated regressions and test for aggregation bias, JASA, 57 (1962), 348-368.

Received: November 12, 2005 